\title{
540 BASELINE MTOR TRANSCRIPTIONAL SIGNATURES IN CD8 T CELLS ARE ASSOCIATED WITH IMMUNE-RELATED ADVERSE EVENTS BUT NOT ANTI-TUMOR RESPONSES IN PATIENTS RECEIVING IMMUNE CHECKPOINT INHIBITORS
}

${ }^{1}$ Chen Zhao* ${ }^{3}$ Matthew Mule, ${ }^{3}$ Andrew Martins, ${ }^{4}$ lago Pinal Fernandez, ${ }^{2}$ Renee Donahue, ${ }^{3}$ Jinguo Chen, ${ }^{2}$ Jeffrey Schlom, ${ }^{2}$ James Gulley, ${ }^{4}$ Andrew Mammen, ${ }^{3} J o h n$ Tsang, ${ }^{2}$ Arun Rajan. ${ }^{1} \mathrm{NIH}$, North Bethesda, USA; ${ }^{2} \mathrm{NCl}$, North Bethesda, USA; ${ }^{3} \mathrm{NIAID}$, North Bethesda, USA; ${ }^{4}$ NIAMS, North Bethesda, USA

Background Immune checkpoint inhibitors (ICIs) have changed the cancer treatment landscape, but immune-related adverse events (irAEs) can affect a wide range of tissues in patients receiving ICIs. Severe irAEs can be life-threatening or fatal and prohibit patients from receiving further ICI treatment. While the clinical features of irAEs are well documented, the pathological mechanisms and predictive biomarkers are largely unknown. In addition, there is a critical need to preserve ICIinduced anti-tumor immunity while controlling for irAEs, which requires deciphering molecular and cellular signatures associated specifically with irAEs beyond those more generally linked to anti-tumor immunity.

Methods To unbiasedly identify immune cells and states associated with irAEs, we applied CITE-seq to measure transcripts and surface proteins (83 protein markers) from PBMCs collected from patients with thymic epithelial tumors before and after treatment with an anti-PD-L1 antibody (avelumab, NCT01772004, NCT03076554).

Results Samples from 9 patients were analyzed. No patient had a history of pre-existing paraneoplastic autoimmune disease. Anti-tumor activity was observed in all cases, and 5 patients had clinical and/or biochemical evidence of immunerelated muscle inflammation (myositis with or without myocarditis). Multilevel models applied within highly resolved cell clusters revealed transcriptional states associated with ICI response and more uniquely with irAEs. A total of 190,000 cells were included in the analysis after quality control. Most notably, CD45RA+ effector memory CD8 T cells with an mTOR transcriptional signature were highly enriched at baseline and post treatment in patients with irAEs.

Conclusions Our findings suggest the potential therapeutic avenues by using mTOR inhibitors to dampen autoimmune responses while potentially sparing anti-tumor activity, to prevent treatment discontinuation and improve clinical outcomes for cancer patients treated with ICIs.

Acknowledgements This research was supported in part by the Intramural Research Program of the NCI (the Center for Cancer Research), NIAID and NIAMS, and through a Cooperative Research and Development Agreement between the National Cancer Institute and EMD Serono.

Trial Registration NCT01772004, NCT03076554

Ethics Approval This study is approved by NCI institutional review board.

http://dx.doi.org/10.1136/jitc-2021-SITC2021.540 